\title{
On the Mahler measure of hyperelliptic families
}

\author{
Marie José Bertin ${ }^{1}$ • Wadim Zudilin ${ }^{2,3}$
}

Received: 23 February 2016 / Accepted: 4 July 2016 / Published online: 14 July 2016

(C) The Author(s) 2016. This article is published with open access at Springerlink.com

\begin{abstract}
We prove Boyd's "unexpected coincidence" of the Mahler measures for two families of two-variate polynomials defining curves of genus 2 . We further equate the same measures to the Mahler measures of polynomials $y^{3}-y+x^{3}-x+k x y$ whose zero loci define elliptic curves for $k \neq 0, \pm 3$.
\end{abstract}

Keywords Mahler measure $\cdot L$-value $\cdot$ Elliptic curve $\cdot$ Hyperelliptic curve $\cdot$ Elliptic integral

Resumé Nous démontrons "coïncidence inattendue" de Boyd des mesures de Mahler pour deux familles de polynômes à deux variables qui définissent les courbes de genre 2 . En outre, nous assimilons les mêmes mesures pour les mesures de Mahler de polynômes $y^{3}-y+$ $x^{3}-x+k x y$ dont zéro loci définir des courbes elliptiques pour $k \neq 0, \pm 3$.

Mathematics Subject Classification Primary 11F67; Secondary 11F11 - 11F20 - 11G16 . $11 \mathrm{G} 55 \cdot 11 \mathrm{R} 06 \cdot 14 \mathrm{H} 52 \cdot 19 \mathrm{~F} 27$

\section{Introduction}

In his pioneering systematic study [2] of the Mahler measures of two-variate polynomials Boyd has distinguished several special families, for which the measures are related to the

Wadim Zudilin

wzudilin@mpim-bonn.mpg.de

Marie José Bertin

marie-jose.bertin@imj-prg.fr

1 Institut de Mathématiques, Université Pierre et Marie Curie (Paris 6), 4 Place Jussieu, 75252 Paris, France

2 Max-Planck-Institut für Mathematik, Vivatsgasse 7, 53111 Bonn, Germany

3 School of Mathematical and Physical Sciences, The University of Newcastle, Callaghan, NSW 2308, Australia 
$L$-values of the curves defined by the zero loci of the polynomials. The two particular families

$$
P_{k}(x, y)=\left(x^{2}+x+1\right) y^{2}+k x(x+1) y+x\left(x^{2}+x+1\right)
$$

and

$$
Q_{k}(x, y)=\left(x^{2}+x+1\right) y^{2}+\left(x^{4}+k x^{3}+(2 k-4) x^{2}+k x+1\right) y+x^{2}\left(x^{2}+x+1\right)
$$

are nicknamed in [2] as Family 3.2 and Family 3.5B, respectively. Generically, both $P_{k}(x, y)=0$ and $Q_{k}(x, y)=0$ define curves of genus 2 whose jacobians are isogenous to the product of two elliptic curves. Computing the Mahler measures of $P_{k}(x, y)$ and $Q_{k}(x, y)$ numerically and identifying them as rational multiples of the $L$-values $L^{\prime}\left(E_{k}, 0\right)$, where

$$
E_{k}: y^{2}=x^{3}+\left(k^{2}-24\right) x^{2}-16\left(k^{2}-9\right) x
$$

is isomorphic to one of the elliptic curves in the product for each of the two families, Boyd observes the "unexpected coincidence" $\mathrm{m}\left(P_{k}\right)=\mathrm{m}\left(Q_{k+2}\right)$ for integer $k$ in the range $4 \leq$ $k \leq 33$ (but not for $k \leq 3$ ). The primary goal of this note is to confirm Boyd's observation.

Theorem 1 For real $k \geq 4$, we have $\mathrm{m}\left(P_{k}\right)=\mathrm{m}\left(Q_{k+2}\right)$.

Note that for $k \neq 0, \pm 3$ the curve $E_{k}$ is elliptic and it is isomorphic to the elliptic curve $R_{k}(x, y)=0$, where the polynomial

$$
R_{k}(x, y)=y^{3}-y+x^{3}-x+k x y
$$

is tempered-all the faces of its Newton polygon are represented by cyclotomic polynomials. The elliptic origin of the family $R_{k}(x, y)$ and Beilinson's conjectures predict [2,5] that, apart from a finite set of $k$, the measure $\mathrm{m}\left(R_{k}\right)$ is $\mathbb{Q}$-proportional to the $L$-value $L^{\prime}\left(E_{k}, 0\right)$ for $k \in \mathbb{Z}$ (in fact, even for $k$ such that $k^{2} \in \mathbb{Z}$ as in any such case the curve $R_{k}(x, y)=0$ possesses the model defined over $\mathbb{Z}$ ). Our next result unites the predictions with the findings of Boyd in [2].

Theorem 2 For real $k$ satisfying $|k| \geq 16 /(3 \sqrt{3})=3.0792 \ldots$, we have $\mathrm{m}\left(P_{k}\right)=\mathrm{m}\left(R_{k}\right)$.

Noticing that $P_{-k}(x, y)=P_{k}(x,-y)$ and $R_{-k}(x, y)=R_{k}(-x,-y)$ we conclude that $\mathrm{m}\left(P_{|k|}\right)=\mathrm{m}\left(P_{k}\right)$ and $\mathrm{m}\left(R_{|k|}\right)=\mathrm{m}\left(R_{k}\right)$, hence it is sufficient to establish the identity in Theorem 2 and analyse the two polynomial families for positive real $k$ only.

Our analysis of the three polynomial families is performed in Sects. 1-3, each section devoted to one family. We compute the derivatives of the corresponding Mahler measures with respect to the parameter $k$ and make use of the easily seen asymptotics

$$
\mathrm{m}\left(P_{k}\right)=\log |k|+o(1), \quad \mathrm{m}\left(Q_{k}\right)=\log |k|+o(1) \quad \text { and } \quad \mathrm{m}\left(R_{k}\right)=\log |k|+o(1)
$$

as $|k| \rightarrow \infty$, to conclude about the equality of the Mahler measures themselves. This is a strategy we have successfully employed before in [1]. Our findings provide one with the reasons of why the ranges for $k$ in Theorems 1 and 2 cannot be refined, and in Sect. 4 we discuss some further aspects of this "expected noncoincidence."

One of our reasons for linking the Mahler measures of hyperelliptic families $P_{k}(x, y)$ and $Q_{k}(x, y)$ to that of elliptic family $R_{k}(x, y)$, not previously displayed, is a hope to actually prove $\mathrm{m}\left(R_{k}\right)=c_{k} L^{\prime}\left(E_{k}, 0\right)$ with $c_{k} \in \mathbb{Q}^{\times}$for some values of $k$. Armed with the recent formula for the regulator of modular units [7] and its far-going generalisation for the regulator of Siegel units [4] established by Brunault, such identities are expected to be automated in the near future. The main obstacle to produce a single example for $\mathrm{m}\left(R_{k}\right)$ is of purely 
computational nature: the smallest conductor of the elliptic curve $E_{k}$ one gets for $k>3$, $k^{2} \in \mathbb{Z}$, is $224=2^{5} \times 7$ when $k=4$. We further comment on this circumstance and on a related conjecture of Boyd for $\mathrm{m}\left(Q_{-1}\right)$ in the final section.

\section{The first family}

We use the equality $\mathrm{m}\left(P_{|k|}\right)=\mathrm{m}\left(P_{k}\right)$ to reduce our analysis in this section to that for $k \geq 0$.

Write $P_{k}\left(x^{2}, y\right)=x^{4} \widetilde{P}_{k}(x, y / x)$, where

$$
\begin{aligned}
\widetilde{P}_{k}(x, y) & =\left(x^{2}+x^{-2}+1\right) y^{2}+k\left(x+x^{-1}\right) y+\left(x^{2}+x^{-2}+1\right) \\
& =\left(x+x^{-1}+1\right)\left(x+x^{-1}-1\right) y^{2}+k\left(x+x^{-1}\right) y+\left(x+x^{-1}+1\right)\left(x+x^{-1}-1\right) \\
& =\left(x+x^{-1}+1\right)\left(x+x^{-1}-1\right)\left(y-y_{1}(x)\right)\left(y-y_{2}(x)\right)
\end{aligned}
$$

and

$$
\begin{aligned}
\left\{y_{1}(x), y_{2}(x)\right\} & =\frac{-k\left(x+x^{-1}\right) \pm \sqrt{\Delta_{k}(x)}}{2\left(x+x^{-1}+1\right)\left(x+x^{-1}-1\right)} \\
\Delta_{k}(x) & =k^{2}\left(x+x^{-1}\right)^{2}-4\left(\left(x+x^{-1}\right)^{2}-1\right)^{2} .
\end{aligned}
$$

By Viète's theorem $y_{1}(x) y_{2}(x)=1$ implying that $\left|y_{1}(x)\right|=\left|y_{2}(x)\right|=1$ if $\Delta_{k}(x) \leq 0$ and $\left|y_{2}(x)\right|<1<\left|y_{1}(x)\right|$ if $\Delta_{k}(x)>0$, when we order the zeroes $y_{1}(x), y_{2}(x)$ appropriately. In the latter case

$$
\left|y_{1}(x)\right|=\max \left\{\left|y_{1}(x)\right|,\left|y_{2}(x)\right|\right\}=\frac{k\left|x+x^{-1}\right|+\sqrt{\Delta_{k}(x)}}{2\left|\left(x+x^{-1}\right)^{2}-1\right|}>1
$$

and

$$
\left|y_{2}(x)\right|=\min \left\{\left|y_{1}(x)\right|,\left|y_{2}(x)\right|\right\}<1 .
$$

In notation $x=e^{i \theta},-\pi<\theta<\pi$, we let $c=\cos ^{2} \theta$, so that $c$ ranges in [0, 1]. Since $x+x^{-1}=2 \cos \theta$, we get

$$
\begin{aligned}
\Delta_{k} & =4 k^{2} c-4(4 c-1)^{2}=-4\left(16 c^{2}-\left(8+k^{2}\right) c+1\right) \\
& =-64\left(c-c_{-}(k)\right)\left(c-c_{+}(k)\right),
\end{aligned}
$$

where

$$
c_{ \pm}(k)=\frac{8+k^{2} \pm k \sqrt{16+k^{2}}}{32} .
$$

Because $0<c_{-}(k)<c_{+}(k)<1$ for $0<k<3$ and $0<c_{-}(k)<1<c_{+}(k)$ if $k>3$, we have $\Delta_{k} \geq 0$ iff $c_{-}(k) \leq c \leq \min \left\{1, c_{+}(k)\right\}$. Note that

$$
\left|y_{1}(x)\right|=\frac{k \sqrt{c}+4 \sqrt{-\left(c-c_{-}(k)\right)\left(c-c_{+}(k)\right)}}{|4 c-1|} .
$$


Using Jensen's formula and the symmetry $y_{1}(x)=y_{1}\left(x^{-1}\right)$, we obtain

$$
\begin{aligned}
p(k) & =\mathrm{m}\left(P_{k}(x, y)\right)=\mathrm{m}\left(\widetilde{P}_{k}(x, y)\right) \\
& =\frac{1}{(2 \pi i)^{2}} \iint_{|x|=|y|=1} \log \left|\widetilde{P}_{k}(x, y)\right| \frac{\mathrm{d} x}{x} \frac{\mathrm{d} y}{y} \\
& =\frac{1}{2 \pi i} \int_{|x|=1} \log \left|y_{1}(x)\right| \frac{\mathrm{d} x}{x} \\
& =\frac{1}{\pi i} \int_{|x|=1} \operatorname{Re} \log y_{1}(x) \frac{\mathrm{d} x}{x} \\
& =\frac{1}{\pi i} \int_{|x|=1} \operatorname{Re} \log \frac{k\left|x+x^{-1}\right|+\sqrt{\Delta_{k}(x)}}{2\left(x+x^{-1}+1\right)\left(x+x^{-1}-1\right)} \frac{\mathrm{d} x}{x} \\
& =\frac{1}{\pi i} \int_{|x|=1} \operatorname{Re} \log \frac{k\left|x+x^{-1}\right|+\sqrt{\Delta_{k}(x)}}{2} \frac{\mathrm{d} x}{x} \\
& =\frac{1}{\pi} \operatorname{Re} \int_{0}^{\pi} \log \left(k|\cos \theta|+\sqrt{-\left(16 \cos ^{4} \theta-\left(8+k^{2}\right) \cos ^{2} \theta+1\right)}\right) \mathrm{d} \theta, \quad k>0 .
\end{aligned}
$$

The derivative of the result with respect to $k$ is

$$
\begin{aligned}
\frac{\mathrm{d} p(k)}{\mathrm{d} k} & =\frac{1}{\pi} \operatorname{Re} \int_{0}^{\pi} \frac{|\cos \theta|}{\sqrt{-\left(16 \cos ^{4} \theta-\left(8+k^{2}\right) \cos ^{2} \theta+1\right)}} \mathrm{d} \theta \\
& =\frac{1}{\pi} \operatorname{Re} \int_{-1}^{1} \frac{|t|}{\sqrt{-\left(16 t^{4}-\left(8+k^{2}\right) t^{2}+1\right)}} \frac{\mathrm{d} t}{\sqrt{1-t^{2}}} \\
& =\frac{2}{\pi} \operatorname{Re} \int_{0}^{1} \frac{t}{\sqrt{-\left(16 t^{4}-\left(8+k^{2}\right) t^{2}+1\right)}} \frac{\mathrm{d} t}{\sqrt{1-t^{2}}} \\
& =\frac{1}{\pi} \operatorname{Re} \int_{0}^{1} \frac{\mathrm{d} c}{\sqrt{-\left(16 c^{2}-\left(8+k^{2}\right) c+1\right)}} \frac{\mathrm{d} c}{\sqrt{1-c}} \\
& =\frac{1}{4 \pi} \int_{c_{-}(k)}^{\min \left\{1, c_{+}(k)\right\}} \frac{\frac{\sqrt{\left(c-c_{-}(k)\right)\left(c-c_{+}(k)\right)(c-1)}}{\sqrt{(2)}}}{}
\end{aligned}
$$

which is a complete elliptic integral.

Performing additionally the change $c=(4-v) / 16$ we obtain

$$
\begin{aligned}
\frac{\mathrm{d} p(k)}{\mathrm{d} k} & =\frac{1}{\pi} \operatorname{Re} \int_{-12}^{4} \frac{\mathrm{d} v}{\sqrt{-(v+12)\left(v^{2}+k^{2} v-4 k^{2}\right)}} \\
& =\frac{1}{\pi} \int_{\max \left\{-12,-k\left(k+\sqrt{k^{2}+16}\right) / 2\right\}}^{-k\left(k-\sqrt{k^{2}+16}\right) / 2} \frac{\mathrm{d} v}{\sqrt{-(v+12)\left(v^{2}+k^{2} v-4 k^{2}\right)}} ;
\end{aligned}
$$

in particular, we have the following.

Proposition 1 For $k \geq 3$,

$$
\frac{\mathrm{d} p(k)}{\mathrm{d} k}=\frac{1}{\pi} \int_{-12}^{-k\left(k-\sqrt{k^{2}+16}\right) / 2} \frac{\mathrm{d} v}{\sqrt{-(v+12)\left(v^{2}+k^{2} v-4 k^{2}\right)}} .
$$




\section{The second family}

The analysis here is very similar to the one we had in the paper [1]. First introduce $Q_{k+2}(x, y)=x^{3} \widetilde{Q}_{k+2}(x, y / x)$, where

$$
\begin{aligned}
\widetilde{Q}_{k+2}(x, y)= & \left(x+x^{-1}+1\right) y^{2}+\left(x^{2}+x^{-2}+(k+2)\left(x+x^{-1}\right)+2 k\right) y+\left(x+x^{-1}+1\right) \\
= & \left(x+x^{-1}+1\right) y^{2}+\left(\left(x+x^{-1}\right)^{2}+(k+2)\left(x+x^{-1}\right)+2(k-1)\right) y \\
& +\left(x+x^{-1}+1\right) .
\end{aligned}
$$

Write

$$
\widetilde{Q}_{k+2}(x, y)=\left(x+x^{-1}+1\right)\left(y-y_{1}(x)\right)\left(y-y_{2}(x)\right),
$$

where

$$
\left\{y_{1}(x), y_{2}(x)\right\}=\frac{-B_{k}(x) \pm \sqrt{\Delta_{k}(x)}}{2\left(x+x^{-1}+1\right)}
$$

and $B_{k}(x)=\left(x+x^{-1}\right)^{2}+(k+2)\left(x+x^{-1}\right)+2(k-1)$,

$$
\begin{aligned}
\Delta_{k}(x) & =B_{k}(x)^{2}-4\left(x+x^{-1}+1\right)^{2} \\
& =\left(x+x^{-1}+2\right)\left(x+x^{-1}+k-2\right)\left(\left(x+x^{-1}\right)^{2}+(k+4)\left(x+x^{-1}\right)+2 k\right) .
\end{aligned}
$$

By Viète's theorem $y_{1}(x) y_{2}(x)=1$ implying that $\left|y_{1}(x)\right|=\left|y_{2}(x)\right|=1$ if $\Delta_{k}(x) \leq 0$ and $\left|y_{2}(x)\right|<1<\left|y_{1}(x)\right|$ if $\Delta_{k}(x)>0$, when we order the zeroes $y_{1}(x), y_{2}(x)$ appropriately. In the latter case

$$
y_{1}(x)=\frac{-B_{k}(x)-\operatorname{sign}\left(B_{k}(x)\right) \sqrt{\Delta_{k}(x)}}{2\left(x+x^{-1}+1\right)} .
$$

Note that

$$
\begin{aligned}
\frac{\mathrm{d}}{\mathrm{d} k} \log y_{1}(x) & =\frac{\mathrm{d}}{\mathrm{d} k} \log \left(B_{k}(x)+\operatorname{sign}\left(B_{k}(x)\right) \sqrt{B_{k}(x)^{2}-4\left(x+x^{-1}+1\right)^{2}}\right) \\
& =\left.\frac{\mathrm{d}}{\mathrm{d} B} \log \left(B+\operatorname{sign}(B) \sqrt{B^{2}-4\left(x+x^{-1}+1\right)^{2}}\right)\right|_{B=B_{k}(x)} \cdot \frac{\mathrm{d} B_{k}}{\mathrm{~d} k} \\
& =-\frac{\operatorname{sign}\left(B_{k}(x)\right)}{\sqrt{B_{k}(x)^{2}-4\left(x+x^{-1}+1\right)^{2}}} \cdot\left(x+x^{-1}+2\right) .
\end{aligned}
$$

With the help of Jensen's formula we obtain

$$
\begin{aligned}
q(k+2) & =\mathrm{m}\left(Q_{k+2}(x, y)\right)=\mathrm{m}\left(\widetilde{Q}_{k+2}(x, y)\right) \\
& =\frac{1}{(2 \pi i)^{2}} \iint_{|x|=|y|=1} \log \left|\widetilde{Q}_{k+2}(x, y)\right| \frac{\mathrm{d} x}{x} \frac{\mathrm{d} y}{y} \\
& =\frac{1}{2 \pi i} \int_{|x|=1} \log \left|y_{1}(x)\right| \frac{\mathrm{d} x}{x} \\
& =\frac{1}{\pi i} \int_{|x|=1} \operatorname{Re} \log y_{1}(x) \frac{\mathrm{d} x}{x} \\
& =\frac{1}{\pi} \operatorname{Re} \int_{0}^{\pi} \log y_{1}\left(e^{i \theta}\right) \mathrm{d} \theta
\end{aligned}
$$


leading to

$$
\begin{aligned}
\frac{\mathrm{d} q(k+2)}{\mathrm{d} k} & =-\frac{1}{\pi} \operatorname{Re} \int_{0}^{\pi} \frac{\operatorname{sign}\left(B_{k}\left(e^{i \theta}\right)\right)}{\sqrt{\Delta_{k}\left(e^{i \theta}\right)}}(2 \cos \theta+2) \mathrm{d} \theta \\
& =-\frac{1}{\pi} \operatorname{Re} \int_{-1}^{1} \frac{\operatorname{sign}\left(2 t^{2}+(k+2) t+k-1\right)}{\sqrt{4(t+1)(2 t+k-2)\left(2 t^{2}+(k+4) t+k\right)}} \frac{(2 t+2) \mathrm{d} t}{\sqrt{1-t^{2}}} \\
& =-\frac{1}{\pi} \operatorname{Re} \int_{-1}^{1} \frac{\operatorname{sign}((t+1)(2 t+k)-1)}{\sqrt{(1-t)(2 t+k-2)\left(2 t^{2}+(k+4) t+k\right)}} \mathrm{d} t .
\end{aligned}
$$

Note that for $k>0$ we have

$$
\begin{array}{rlrl}
2 \operatorname{Re} \int_{-1}^{1} \operatorname{sign}\left(2 t^{2}+(k+2) t+k-1\right) & =-\int_{-1}^{\left(-k-4+\sqrt{16+k^{2}}\right) / 4}+\int_{1-k / 2}^{1} & & \text { if } \quad 0<k \leq 3, \\
& =-\int_{-1}^{1-k / 2}+\int_{\left(-k-4+\sqrt{16+k^{2}}\right) / 4}^{1} & & \text { if } 3<k<4, \\
& =\int_{\left(-k-4+\sqrt{16+k^{2}}\right) / 4}^{1} & \text { if } k \geq 4 .
\end{array}
$$

Performing the change of variable $t=(v+2 k(k+1)) /(v-4 k)$ we then obtain

$$
\frac{\mathrm{d} q(k+2)}{\mathrm{d} k}=\frac{1}{\pi}\left(\int_{-\infty}^{-12}-\int_{-k\left(k+\sqrt{16+k^{2}}\right) / 2}^{k(1-k)}\right) \frac{\mathrm{d} v}{\sqrt{-(v+12)\left(v^{2}+k^{2} v-4 k^{2}\right)}}
$$

if $0<k \leq 3$,

$$
\frac{\mathrm{d} q(k+2)}{\mathrm{d} k}=\frac{1}{\pi}\left(\int_{-\infty}^{-k\left(k+\sqrt{16+k^{2}}\right) / 2}-\int_{-12}^{k(1-k)}\right) \frac{\mathrm{d} v}{\sqrt{-(v+12)\left(v^{2}+k^{2} v-4 k^{2}\right)}}
$$

if $3<k<4$, and

$$
\frac{\mathrm{d} q(k+2)}{\mathrm{d} k}=\frac{1}{\pi} \int_{-\infty}^{-k\left(k+\sqrt{16+k^{2}}\right) / 2} \frac{\mathrm{d} v}{\sqrt{-(v+12)\left(v^{2}+k^{2} v-4 k^{2}\right)}}
$$

if $k \geq 4$.

Remark 1 The appearance of incomplete elliptic integrals

$$
\int_{-k\left(k+\sqrt{16+k^{2}}\right) / 2}^{k(1-k)} \frac{\mathrm{d} v}{\sqrt{-(v+12)\left(v^{2}+k^{2} v-4 k^{2}\right)}}
$$

and

$$
\int_{-12}^{k(1-k)} \frac{\mathrm{d} v}{\sqrt{-(v+12)\left(v^{2}+k^{2} v-4 k^{2}\right)}}
$$

for $k<4$ hints on why the Mahler measures $q(k+2)$ are possibly not related to the corresponding $L$-values (see the question marks and the "half-Mahler" measures $\mathrm{m}^{\prime}$ in [2, Table 9]). Our next statement refers to the situation when incomplete elliptic integrals do not occur.

Proposition 2 For $k \geq 4$,

$$
\frac{\mathrm{d} p(k)}{\mathrm{d} k}=\frac{\mathrm{d} q(k+2)}{\mathrm{d} k} .
$$


Proof We will show that

$$
\begin{aligned}
& \int_{-12}^{-k\left(k-\sqrt{16+k^{2}}\right) / 2} \frac{\mathrm{d} v}{\sqrt{-(v+12)\left(v^{2}+k^{2} v-4 k^{2}\right)}} \\
& =\int_{-\infty}^{-k\left(k+\sqrt{16+k^{2}}\right) / 2} \frac{\mathrm{d} v}{\sqrt{-(v+12)\left(v^{2}+k^{2} v-4 k^{2}\right)}}
\end{aligned}
$$

for $k>3$. On comparing the integrals in (3) and (4) this implies the required coincidence.

The involution

$$
v \mapsto-\frac{4\left(3 v+4 k^{2}\right)}{v+12}
$$

interchanges $\infty$ with -12 and $-k\left(k+\sqrt{k^{2}+16}\right) / 2$ with $-k\left(k-\sqrt{k^{2}+16}\right) / 2$. Applying the change to one of the integrals in (5) we arrive at the other.

Proof of Theorem 1 Proposition 2 implies that $p(k)=q(k+2)+C$ for $k \geq 4$, with some constant $C$ independent of $k$. On using the asymptotics (2) we conclude that $C=0$, and the theorem follows.

\section{The third family}

Since $\mathrm{m}\left(R_{|k|}\right)=\mathrm{m}\left(R_{k}\right)$, we assume that $k \geq 0$ throughout the section.

For the elliptic family we write

$$
-y^{3} R_{k}(x / y, 1 /(x y))=\widetilde{R}_{k}(x, y)=\left(x+x^{-1}\right) y^{2}-k y-\left(x^{3}+x^{-3}\right) .
$$

This time the zeroes $y_{1}(x)$ and $y_{2}(x)$ of the quadratic polynomial $\widetilde{R}_{k}(x, y)$ satisfy

$$
y_{1}(x) y_{2}(x)=-\frac{x^{3}+x^{-3}}{x+x^{-1}}=-\left(x^{2}-1+x^{-2}\right)=3-4 \cos ^{2} \theta .
$$

We have

$$
\begin{aligned}
& y_{1}(x)=\frac{k+\sqrt{k^{2}-16 \cos ^{2} \theta\left(3-4 \cos ^{2} \theta\right)}}{4 \cos \theta}, \\
& y_{2}(x)=\frac{k-\sqrt{k^{2}-16 \cos ^{2} \theta\left(3-4 \cos ^{2} \theta\right)}}{4 \cos \theta},
\end{aligned}
$$

so that $\left|y_{1}(x)\right| \geq\left|y_{2}(x)\right|$.

Lemma 1 If $k \geq 3$ then $\Delta_{k}(x) \geq 0$, so that both $y_{1}(x)$ and $y_{2}(x)$ are real. If $0 \leq k<3$ then $y_{1}(x)$ and $y_{2}(x)$ are complex conjugate to each other for

$$
\frac{3-\sqrt{9-k^{2}}}{8}<\cos ^{2} \theta<\frac{3+\sqrt{9-k^{2}}}{8},
$$

so that $\left|y_{1}(x)\right|=\left|y_{2}(x)\right|=\left|3-4 \cos ^{2} \theta\right|^{1 / 2}$ in this case. Furthermore, $\left|y_{1}(x)\right|=\left|y_{2}(x)\right|>1$ if and only if

$$
\begin{array}{ll}
\frac{3-\sqrt{9-k^{2}}}{8}<\cos ^{2} \theta<\frac{1}{2} & \text { for } 0 \leq k<2 \sqrt{2}, \\
\frac{3-\sqrt{9-k^{2}}}{8}<\cos ^{2} \theta<\frac{3+\sqrt{9-k^{2}}}{8} & \text { for } 2 \sqrt{2} \leq k<3 .
\end{array}
$$


Proof Note that $16 \cos ^{2} \theta\left(3-4 \cos ^{2} \theta\right) \leq \max _{0 \leq c \leq 1} 16 c(3-4 c)=9$, hence

$$
\Delta_{k}(x)=k^{2}-16 \cos ^{2} \theta\left(3-4 \cos ^{2} \theta\right) \geq 0 \quad \text { if } \quad k \geq 3 .
$$

The second part of the statement is a mere computation.

Lemma 2 If $k \geq 2 \sqrt{2}$ then $\left|y_{1}(x)\right| \geq 1$ for all $x \in \mathbb{C}:|x|=1$.

Proof Denote $c=\cos ^{2} \theta$ for $x=\exp (i \theta)$, so that our task is to show that

$$
\left|k+\sqrt{k^{2}-48 c+64 c^{2}}\right| \geq 4 \sqrt{c}
$$

for $0 \leq c \leq 1$. If $k^{2}-48 c+64 c^{2} \geq 0$, meaning that either $k \geq 3$ and $c \in[0,1]$ or $2 \sqrt{2} \leq k<3$ and $c \in\left[0,\left(3-\sqrt{9-k^{2}}\right) / 8\right] \cup\left[\left(3+\sqrt{9-k^{2}}\right) / 8,1\right]$, the inequality $(6)$ is equivalent to

$$
\sqrt{k^{2}-48 c+64 c^{2}} \geq 4 \sqrt{c}-k .
$$

The latter inequality holds automatically when the right-hand side is nonpositive, that is, when $c \leq k^{2} / 16$. If $c>k^{2} / 16 \geq 1 / 2$ then

$$
\sqrt{c}(1-c) \leq \frac{k}{4}\left(1-\frac{k^{2}}{16}\right)<\frac{k}{4} \cdot \frac{1}{2}=\frac{k}{8}
$$

implying that $k^{2}-48 c+64 c^{2}<(4 \sqrt{c}-k)^{2}=k^{2}-8 k \sqrt{c}+16 c$, and the required inequality follows.

$$
\text { If } k^{2}-48 c+64 c^{2}<0 \text { then }\left|y_{1}(x)\right|=\left|y_{2}(x)\right|=\left|y_{1}(x) y_{2}(x)\right|^{1 / 2} \text { and }
$$

$$
\left|k+\sqrt{k^{2}-48 c+64 c^{2}}\right|=|3-4 c|^{1 / 2} .
$$

The latter expression is $\geq 1$ whenever $0 \leq c \leq 1 / 2$; this indeed holds true for $(3-$ $\left.\sqrt{9-k^{2}}\right) / 8<c<\left(3+\sqrt{9-k^{2}}\right) / 8$ since $2 \sqrt{2} \leq \bar{k} \leq 3$ in this case.

The required inequality (6) is thus established.

Lemma 3 If $k \geq 16 /(3 \sqrt{3})=3.0792 \ldots$ then $\left|y_{2}(x)\right| \leq 1$ for all $x \in \mathbb{C}:|x|=1$.

Proof To verify that $k-\sqrt{k^{2}-48 c+64 c^{2}} \leq 4 \sqrt{c}$, equivalently

$$
\sqrt{k^{2}-48 c+64 c^{2}} \geq k-4 \sqrt{c}
$$

for $0 \leq c \leq 1$, we first notice that the inequality is trivially true for $c \geq k^{2} / 16$ since the right-hand side is then nonpositive. If $c<k^{2} / 16$, the inequality (7) after squaring becomes equivalent to $8 \sqrt{c}(1-c) \leq k$. The latter inequality holds true because the maximum of $\sqrt{c}(1-c)$ is attained at $c=1 / 3$ and is equal to $2 /(3 \sqrt{3})$.

Proposition 3 If $k \geq 16 /(3 \sqrt{3})$ then

$$
\frac{\mathrm{d} r(k)}{\mathrm{d} k}=\frac{1}{\pi} \int_{0}^{1} \frac{\mathrm{d} c}{\sqrt{c(1-c)\left(k^{2}-48 c+64 c^{2}\right)}} .
$$


Proof Using the two lemmas above we conclude that for values of $k \geq 16 /(3 \sqrt{3})$ Jensen's formula gives us

$$
\begin{aligned}
r(k) & =\mathrm{m}\left(R_{k}(x, y)\right)=\mathrm{m}\left(\widetilde{R}_{k}(x, y)\right)=\frac{1}{2 \pi i} \int_{|x|=1} \log \left|y_{1}(x)\right| \frac{\mathrm{d} x}{x} \\
& =\operatorname{Re}\left(\frac{1}{2 \pi i} \int_{|x|=1} \log \frac{k+\sqrt{k^{2}+4\left(x+x^{-1}\right)\left(x^{3}+x^{-3}\right)}}{2} \frac{\mathrm{d} x}{x}\right)-\mathrm{m}\left(x+x^{-1}\right) \\
& =\frac{1}{2 \pi} \operatorname{Re} \int_{-\pi}^{\pi} \log \frac{k+\sqrt{k^{2}-16 \cos ^{2} \theta\left(3-4 \cos ^{2} \theta\right)}}{2} \mathrm{~d} \theta \\
& =\frac{2}{\pi} \operatorname{Re} \int_{0}^{\pi / 2} \log \frac{k+\sqrt{k^{2}-16 \cos ^{2} \theta\left(3-4 \cos ^{2} \theta\right)}}{2} \mathrm{~d} \theta \\
& =\frac{2}{\pi} \operatorname{Re} \int_{0}^{1} \log \frac{k+\sqrt{k^{2}-16 t^{2}\left(3-4 t^{2}\right)}}{2} \frac{\mathrm{d} t}{\sqrt{1-t^{2}}}
\end{aligned}
$$

which in turn implies that

$$
\begin{aligned}
\frac{\mathrm{d} r(k)}{\mathrm{d} k} & =\frac{2}{\pi} \operatorname{Re} \int_{0}^{1} \frac{1}{\sqrt{k^{2}-16 t^{2}\left(3-4 t^{2}\right)}} \frac{\mathrm{d} t}{\sqrt{1-t^{2}}} \\
& =\frac{2}{\pi} \int_{0}^{1} \frac{1}{\sqrt{k^{2}-16 t^{2}\left(3-4 t^{2}\right)}} \frac{\mathrm{d} t}{\sqrt{1-t^{2}}} .
\end{aligned}
$$

It remains to perform the change $c=t^{2}$.

If $0<k<16 /(3 \sqrt{3})$ then the cubic polynomial $f(t)=8 t^{3}-8 t+k$ has two real zeroes on the interval $0<t<1$, since $f(0)=f(1)=k>0$ and $f(1 / \sqrt{3})=k-16 /(3 \sqrt{3})<0$. Denote them $t_{1}(k)<t_{2}(k)$.

Lemma 4 If $|\cos \theta|=\left|x+x^{-1}\right| / 2=t_{1}(k)$ then $\left|y_{2}(x)\right|=1$ for $k \leq 16 /(3 \sqrt{3})$.

If $|\cos \theta|=\left|x+x^{-1}\right| / 2=t_{2}(k)$ then

$$
\left|y_{1}(x)\right|=1 \text { for } 0<k \leq 2 \sqrt{2} \text { and }\left|y_{2}(x)\right|=1 \text { for } 2 \sqrt{2} \leq k \leq 16 /(3 \sqrt{3}) .
$$

Proof Note that for the values of $x$ corresponding to $t_{1}(k)$ and $t_{2}(k)$ we always have $\Delta_{k}(x) \geq$ 0 , so that both $y_{1}(x)$ and $y_{2}(x)$ are real. The solutions of $\left|y_{1}(x)\right|=1$ and $\left|y_{2}(x)\right|=1$ correspond to solving

$$
k \pm \sqrt{k^{2}-16 t^{2}\left(3-4 t^{2}\right)}=4 t,
$$

where $t=|\cos \theta|=\left|x+x^{-1}\right| / 2$. By elementary manipulations the latter equation reduces to $8 t^{3}-8 t+k=0$, and the remaining task is to distinguish whether we get $\left|y_{1}(x)\right|=1$ or $\left|y_{2}(x)\right|=1$. We do not reproduce this technical but elementary analysis here.

Proposition 4 If $0<k<16 /(3 \sqrt{3})$ then

$$
\frac{\mathrm{d} r(k)}{\mathrm{d} k}=\frac{1}{\pi}\left(\int_{0}^{t_{1}(k)^{2}}+\int_{t_{2}(k)^{2}}^{1}\right) \frac{\mathrm{d} c}{\sqrt{c(1-c)\left(k^{2}-48 c+64 c^{2}\right)}},
$$

where $t_{1}(k)$ and $t_{2}(k), 0<t_{1}(k)<1 / \sqrt{3}<t_{2}(k)<1$, are the real zeroes of the polynomial $8 t^{3}-8 t+k$. 
Proof To each $x$ on the unit circle we assign the real parameter $\theta$ such that $x=e^{i \theta}$ and real parameter $t=\left|x+x^{-1}\right| / 2=|\cos \theta| \in[0,1]$. The analysis of Lemmas $1-4$ shows that the ranges of $t$ that correspond to $\left|y_{1}(x)\right| \geq 1$ and $\left|y_{2}(x)\right| \geq 1$ are as follows: if $0<k<2 \sqrt{2}$ then

$\left|y_{1}(x)\right| \geq 1$ for $t \in[0,1 / \sqrt{2}] \cup\left[t_{2}(k), 1\right] \quad$ and $\quad\left|y_{2}(x)\right| \geq 1$ for $t \in\left[t_{1}(k), 1 / \sqrt{2}\right]$; and if $2 \sqrt{2} \leq k<16 /(3 \sqrt{3})$ then

$$
\left|y_{1}(x)\right| \geq 1 \text { for } t \in[0,1] \quad \text { and } \quad\left|y_{2}(x)\right| \geq 1 \text { for } t \in\left[t_{1}(k), t_{2}(k)\right] .
$$

Therefore,

$$
\begin{aligned}
r(k)= & \frac{1}{2 \pi i} \int_{|x|=1} \log \max \left\{\left|y_{1}(x)\right|, 1\right\} \frac{\mathrm{d} x}{x}+\frac{1}{2 \pi i} \int_{|x|=1} \log \max \left\{\left|y_{2}(x)\right|, 1\right\} \frac{\mathrm{d} x}{x} \\
= & \frac{2}{\pi} \operatorname{Re}\left(\int_{0}^{1 / \sqrt{2}}+\int_{t_{2}(k)}^{1}\right) \log \frac{k+\sqrt{k^{2}-16 t^{2}\left(3-4 t^{2}\right)}}{4 t} \frac{\mathrm{d} t}{\sqrt{1-t^{2}}} \\
& +\frac{2}{\pi} \operatorname{Re} \int_{t_{1}(k)}^{1 / \sqrt{2}} \log \frac{k-\sqrt{k^{2}-16 t^{2}\left(3-4 t^{2}\right)}}{4 t} \frac{\mathrm{d} t}{\sqrt{1-t^{2}}}
\end{aligned}
$$

if $0<k<2 \sqrt{2}$ and

$$
\begin{aligned}
= & \frac{2}{\pi} \operatorname{Re} \int_{0}^{1} \log \frac{k+\sqrt{k^{2}-16 t^{2}\left(3-4 t^{2}\right)}}{4 t} \frac{\mathrm{d} t}{\sqrt{1-t^{2}}} \\
& +\frac{2}{\pi} \operatorname{Re} \int_{t_{1}(k)}^{t_{2}(k)} \log \frac{k-\sqrt{k^{2}-16 t^{2}\left(3-4 t^{2}\right)}}{4 t} \frac{\mathrm{d} t}{\sqrt{1-t^{2}}}
\end{aligned}
$$

if $2 \sqrt{2} \leq k<16 /(3 \sqrt{3})$. Differentiating $r(k)$ we obtain

$$
\frac{\mathrm{d} r(k)}{\mathrm{d} k}=\frac{2}{\pi} \operatorname{Re}\left(\int_{0}^{1 / \sqrt{2}}+\int_{t_{2}(k)}^{1}-\int_{t_{1}(k)}^{1 / \sqrt{2}}\right) \frac{1}{\sqrt{k^{2}-16 t^{2}\left(3-4 t^{2}\right)}} \frac{\mathrm{d} t}{\sqrt{1-t^{2}}}
$$

if $0<k<2 \sqrt{2}$ and

$$
=\frac{2}{\pi} \operatorname{Re}\left(\int_{0}^{1}-\int_{t_{1}(k)}^{t_{2}(k)}\right) \frac{1}{\sqrt{k^{2}-16 t^{2}\left(3-4 t^{2}\right)}} \frac{\mathrm{d} t}{\sqrt{1-t^{2}}}
$$

if $2 \sqrt{2} \leq k<16 /(3 \sqrt{3})$; here we have observed that the additionally occurring integrals in the process of differentiating vanish because Re $\log y_{j}(x)=\log \left|y_{j}(x)\right|=0$ by Lemma 4 in the corresponding cases.

Note that for both $0<k<2 \sqrt{2}$ and $2 \sqrt{2} \leq k<16 /(3 \sqrt{3})$ the result is the same:

$$
\frac{\mathrm{d} r(k)}{\mathrm{d} k}=\frac{2}{\pi} \operatorname{Re}\left(\int_{0}^{t_{1}(k)}+\int_{t_{2}(k)}^{1}\right) \frac{1}{\sqrt{k^{2}-16 t^{2}\left(3-4 t^{2}\right)}} \frac{\mathrm{d} t}{\sqrt{1-t^{2}}} .
$$

To complete the proof we apply the substitution $t^{2}=c$.

Remark 2 The integral in (8) is elliptic, while the integrals in (9) are incomplete elliptic: the "completion" of the integrals will require integrating along $c \in\left(0,\left(3-\sqrt{9-k^{2}}\right) / 8\right) \cup$ $\left(\left(3+\sqrt{9-k^{2}}\right) / 8,1\right)$ if $0<k<3$ or $c \in(0,1)$ if $3 \leq k<16 /(3 \sqrt{3})$ rather than along $c \in\left(0, t_{1}(k)^{2}\right) \cup\left(t_{2}(k)^{2}, 1\right)$. The incompleteness serves as a reason for the Mahler measure $r(k)$ not to be rationally related to $L^{\prime}\left(E_{k}, 0\right)$ for $|k|<16 /(3 \sqrt{3})$. 
Proposition 5 For $k$ positive real, $k \neq 3$,

$$
\int_{0}^{1} \frac{\mathrm{d} c}{\sqrt{c(1-c)\left(64 c^{2}-48 c+k^{2}\right)}}=\int_{-12}^{-k\left(k-\sqrt{16+k^{2}}\right) / 2} \frac{\mathrm{d} v}{\frac{\sqrt{-(v+12)\left(v^{2}+k^{2} v-4 k^{2}\right)}}{} .}
$$

Proof Applying the substitution

$$
c=\frac{k(1+t)}{k+\sqrt{k^{2}+16}+\left(k-\sqrt{k^{2}+16}\right) t}
$$

to the integral on the left-hand side we obtain

$$
\begin{aligned}
& \int_{0}^{1} \frac{\mathrm{d} c}{\sqrt{c(1-c)\left(64 c^{2}-48 c+k^{2}\right)}} \\
& \quad=\sqrt{2} \int_{-1}^{1} \frac{\mathrm{d} t}{\sqrt{\left(1-t^{2}\right)\left(k^{2}-24+k \sqrt{k^{2}+16}+\left(-k^{2}+24+k \sqrt{k^{2}+16}\right) t^{2}\right)}} \\
& \quad=2 \sqrt{2} \int_{0}^{1} \frac{\mathrm{d} t}{\left.\sqrt{\left(1-t^{2}\right)\left(k^{2}-24+k \sqrt{k^{2}+16}+\left(-k^{2}+24+k \sqrt{k^{2}+16}\right) t^{2}\right.}\right)}
\end{aligned}
$$

(after the change $u=t^{2}$ )

$$
=\sqrt{2} \int_{0}^{1} \frac{\mathrm{d} u}{\sqrt{u(1-u)\left(k^{2}-24+k \sqrt{k^{2}+16}+\left(-k^{2}+24+k \sqrt{k^{2}+16}\right) u\right)}} .
$$

Now the substitution

$$
u=\frac{2(v+12)}{-k^{2}+24+k \sqrt{k^{2}+16}}
$$

into the latter integral results in the the right-hand side in (10).

Remark 3 For $k>0, k \neq 3$, the identity in Proposition 5 relates the periods of the elliptic curves $E_{k}$ in (1) (which is isomorphic to $u^{2}=(v+12)\left(v^{2}+k^{2} v-4 k^{2}\right)$ ) and

$$
\widehat{E}_{k}: d^{2}=c(1-c)\left(64 c^{2}-48 c+k^{2}\right) .
$$

The curves $E_{k}$ and $\widehat{E}_{k}$ are not isomorphic but the latter one happens to be a quadratic twist of the former.

Proof of Theorem 2 The equality of elliptic integrals in (10) means that the derivatives of $p(k)$ and $r(k)$ coincide for $k \geq 16 /(3 \sqrt{3})$. Thus $p(k)=r(k)+C$ for the range of $k$, and the asymptotics (2) implies that $C=0$ and finishes the proof of the theorem.

\section{Accurateness of Theorem 2 and related comments}

Though our Remarks 1 and 2 are aimed at explaining the choice of ranges for $k$ in Theorems 1 and 2 , in conclusion we would like to specifically address the difference between $\mathrm{m}\left(P_{3}\right)$ and $\mathrm{m}\left(R_{3}\right)$. The choice $k=3$ corresponds to a simultaneous degeneration in the families of curves $P_{k}(x, y)=0$ and $R_{k}(x, y)=0$. 
The curve

$$
P_{3}(x, y)=\left(x^{2}+x+1\right) y^{2}+3 x(x+1) y+x\left(x^{2}+x+1\right)=0
$$

has genus 1 ; it is isomorphic to the conductor 15 elliptic curve $y^{2}+x y+y=x^{3}+x^{2}$ which has Cremona label 15a8 [6, Curve15.a7]. The proof of the evaluation

$$
\mathrm{m}\left(P_{3}\right)=\frac{1}{6} L^{\prime}(\chi-15,-1)=0.99905183 \ldots
$$

was given in [3, Example 3] (by two different methods!).

On the other hand,

$$
R_{3}(x, y)=(x+y-1)\left(x^{2}-x y+y^{2}+x+y\right)
$$

so that

$$
\begin{aligned}
\mathrm{m}\left(R_{3}\right) & =\mathrm{m}(x+y-1)+\mathrm{m}\left(x^{2}-x y+y^{2}+x+y\right) \\
& =L^{\prime}\left(\chi_{-3},-1\right)+\mathrm{m}\left(x^{2}-x y+y^{2}+x+y\right) .
\end{aligned}
$$

Following the technology and notation in [3] to compute the Mahler measure of $A(x, y)=$ $x^{2}-x y+y^{2}+x+y$, we first fix the rational parametrisation

$$
x=\frac{t-2}{t^{2}-t+1}, \quad y=\frac{-t-1}{t^{2}-t+1},
$$

and compute the resultant of $A(x, y)$ and $A^{*}(x, y)=x^{2} y^{2} A(1 / x, 1 / y)$ :

$$
\operatorname{Res}_{y}\left(A, A^{*}\right)=3 x^{2}\left(x^{4}+x^{3}-x^{2}+x+1\right) .
$$

The quartic polynomial has exactly two complex conjugate zeroes

$$
x_{1}=\frac{3+i \sqrt{5+2 \sqrt{13}}}{1+\sqrt{13}}
$$

and $x_{1}^{-1}$ of absolute value 1 . The corresponding values of $y$ satisfying $|y|=1$ and $A(x, y)=0$ are $y=y_{1}=x_{1}^{-1}$ for $x=x_{1}$ and $y=x_{1}$ for $x=x_{1}^{-1}$. The pair $\left(x_{1}, y_{1}\right)$ is generated by

$$
t_{1}=\frac{1-i \sqrt{5+2 \sqrt{13}}}{2} \text {. }
$$

Note that in this case

$$
\begin{aligned}
\eta(x, y) & =\eta\left(\frac{t-2}{t^{2}-t+1}, \frac{-t-1}{t^{2}-t+1}\right) \\
& =\mathrm{d} D\left(-\left[\frac{t+1}{3}\right]+2\left[\frac{t+1}{\zeta_{6}+1}\right]+2\left[\frac{t+1}{\zeta_{6}^{-1}+1}\right]\right),
\end{aligned}
$$

where the 1-form $\eta(g, h)=\log |g| \mathrm{d} \arg h-\log |h| \mathrm{d} \arg g$ is attached to rational nonconstant functions $g$ and $h$ and

$$
D(z)=\operatorname{Im} \sum_{n=1}^{\infty} \frac{z^{n}}{n^{2}}+\arg (1-z) \log |z|
$$


denotes the Bloch-Wigner dilogarithm. Then by results in [3] the Mahler measure of $A(x, y)$ is equal to

$$
\mathrm{m}(A)=\frac{1}{\pi}\left(D\left(\frac{t_{1}+1}{3}\right)-2 D\left(\frac{t_{1}+1}{\zeta_{6}+1}\right)-2 D\left(\frac{t_{1}+1}{\zeta_{6}^{-1}+1}\right)\right)=0.68844794 \ldots
$$

The resulting measure $\mathrm{m}\left(R_{3}\right)=1.01151388 \ldots$ visually appears to be different from (11) confirming that $\mathrm{m}\left(P_{k}\right) \neq \mathrm{m}\left(R_{k}\right)$ at least for $k=3$. Furthermore, $\mathrm{m}\left(R_{3}\right)$ does not seem to be a $\mathbb{Q}$-linear combination of $L^{\prime}\left(\chi_{-3},-1\right)$ and $L^{\prime}\left(\chi_{-15},-1\right)$.

It would be interesting to establish the expected evaluation $\mathrm{m}\left(R_{4}\right)=-\frac{1}{3} L^{\prime}\left(E_{224 \mathrm{a}}, 0\right)$, hence also for $\mathrm{m}\left(P_{4}\right)$ and $\mathrm{m}\left(Q_{6}\right)$, by using the recent formula of Brunault [4] for the regulator of Siegel units. Note that the elliptic curve $R_{4}(x, y)=0$ does not possess a modular-unit parametrisation (so that the formula from [7] is not applicable) and it is isomorphic to the curve $y^{2}=x^{3}+x^{2}-8 x-8$ which has Cremona label 224 a2 [6, Curve224.a1].

Another related conjecture of Boyd [2, Eq. (3-12)] states that

$$
\mathrm{m}\left(Q_{-1}\right)=\frac{1}{3} L^{\prime}\left(\chi_{-7},-1\right)+\frac{1}{6} L^{\prime}\left(\chi_{-15},-1\right)=\frac{7 \sqrt{7}}{12 \pi} L\left(\chi_{-7}, 2\right)+\frac{5 \sqrt{15}}{8 \pi} L\left(\chi_{-15}, 2\right) .
$$

Here $Q_{-1}(x, y)=0$ is an elliptic curve of conductor $210=2 \times 3 \times 5 \times 7$, which is isomorphic to $y^{2}+x y=x^{3}+x^{2}-3 x-3$ with Cremona label 210 d1 [6, Curve210.a3]. Numerics indicates the lack of a modular-unit parametrisation in this case, though a suitable parametrisation by Siegel units and the principal result from [4] are expected to confirm Boyd's observation for $\mathrm{m}\left(Q_{-1}\right)$.

Acknowledgements Open access funding provided by Max Planck Society. We owe our gratitude to François Brunault for helpful comments and discussions on a preliminary version of the note. We thank the anonymous referee for pointing out some typos in an earlier version. A part of the work was done during the authors' visit in the Centre de recherches mathématiques, Université de Montréal, and the second author's stay at the Max-Planck-Institut für Mathematik, Bonn, in Spring 2015. We thank the staff of the institutes for the excellent conditions we experienced when conducting this research. The second author acknowledges the support of the Max-Planck-Institut für Mathematik and the Australian Research Council.

Open Access This article is distributed under the terms of the Creative Commons Attribution 4.0 International License (http://creativecommons.org/licenses/by/4.0/), which permits unrestricted use, distribution, and reproduction in any medium, provided you give appropriate credit to the original author(s) and the source, provide a link to the Creative Commons license, and indicate if changes were made.

\section{References}

1. Bertin, M.J., Zudilin, W.: On the Mahler measure of a family of genus 2 curves. Math. Zeitschrift. 283(3), 1185-1193. doi:10.1007/s00209-016-1637-6

2. Boyd, D.: Mahler's measure and special values of $L$-functions. Exp. Math. 7(1), 37-82 (1998)

3. Boyd, D.W., Rodriguez-Villegas, F.: With an appendix by N. M. Dunfield, Mahler's measure and the dilogarithm (II), Preprint (2003). arXiv:math/0308041 [math.NT]

4. Brunault, F.: Regulators of Siegel units and applications. J. Number Theory 163, 542-569 (2016)

5. Rodriguez-Villegas, F.: Modular Mahler measures I. In: Topics in number theory, (University Park, PA, 1997), Math. Appl., vol. 467, pp. 17-48. Kluwer Acad. Publ, Dordrecht (1999)

6. The LMFDB Collaboration: The L-functions and Modular Forms Database, http://www.lmfdb.org. Accessed 23 December 2015 (2013-2015)

7. Zudilin, W.: Regulator of modular units and Mahler measures. Math. Proc. Camb. Phil. Soc. 156(2), 313-326 (2014) 